\section{Multicenter Experience with Stenting for Symptomatic Carotid Web}

\author{
Diogo C. Haussen ${ }^{a}$ Jonathan A. Grossberg ${ }^{a}$ Sebastian Koch ${ }^{b}$ \\ Amer Malik $^{b}$ Dileep Yavagal ${ }^{b}$ Benjamin Gory ${ }^{c}$ Wolfgang Leesch ${ }^{d}$ \\ Ameer E. Hassan ${ }^{2}$ Anne-Laure Derelle ${ }^{c}$ Sébastien Richard ${ }^{c}$ \\ Clara Barreira $^{a}$ Gustavo Pradilla ${ }^{a}$ Raul G. Nogueira ${ }^{a}$ \\ a Emory University/Grady Memorial Hospital, Atlanta, GA, USA; b University of Miami/ \\ Jackson Memorial Hospital, Miami, FL, USA; ${ }^{\circ}$ Centre Hospitalier Régional Universitaire \\ de Nancy, Nancy, France; ${ }^{d}$ Riverside Neurovascular Specialists, Newport News, VA, USA; \\ eValley Baptist Medical Center, Harlingen, TX, USA
}

\section{Keywords}

Fibromuscular dysplasia $\cdot$ Carotid web $\cdot$ Stroke $\cdot$ Stent

\begin{abstract}
Background: A carotid web (CaW) is a shelf-like lesion in the posterior aspect of the internal carotid bulb and represents an intimal variant of fibromuscular dysplasia. CaW has been associated with recurrent strokes and conventionally treated with surgical excision. We report a multicenter experience of stenting in patients with symptomatic CaWs. Methods: Retrospective review of consecutive patients admitted to 5 comprehensive stroke centers who were identified to have a symptomatic CaW and treated with carotid stenting. A symptomatic CaW was defined by the presence of a shelf-like/linear, smooth filling defect in the posterior aspect of the carotid bulb diagnosed by neck CT angiography (CTA) and confirmed with conventional angiography in patients with negative stroke workup. Results: Twenty-four patients with stented symptomatic CaW were identified (stroke in $83 \%$ and transient ischemic attack in 17\%). Their median age was 47 years (IQR 41-61), 14 (58\%) were female, and were 17 (71\%) black. The degree of stenosis by NASCET was $0 \%$ (range $0-11$ ). All patients were placed on dual antiplatelets and stented at a median of 9 days (IQR 4-35) after the last event. Closedcell stents were used in 18 (75\%) of the cases. No periprocedural events occurred with the exception of 2 cases of asymptomatic hypotension/bradycardia. Clinical follow-up after stent placement occurred for a median of 12 months (IQR 3-19) with no new cerebrovascular events noted. Functional independence at 90 days was achieved in 22 (91\%) patients. Follow-up vascular imaging (ultrasound $n=18 / \mathrm{CTA} n=5$ ) was performed at a median of 10 months (IQR 3-18) and revealed no stenosis. Conclusions: Stenting for symptomatic CaW appears to be a safe and effective alternative to surgical resection. Further studies are warranted.
\end{abstract}




\section{Introduction}

Carotid web ( $\mathrm{CaW}$ ) is an intimal variant of fibromuscular dysplasia with a specific phenotypic expression as a focal shelf-like lesion in the posterior aspect of the internal carotid bulb from fibrotic dysplasia/hyperplasia [1, 2]. CaW has been associated with recurrent strokes, typically involving the young, and being frequently refractory to standard antiplatelet therapy [1]. Treatment with surgical excision has been performed with encouraging results $[1,2]$. Carotid artery stenting (CAS) for CaW has been rarely described [3-6]. We aim to report a multicenter experience of CAS in patients with symptomatic $\mathrm{CaW}$, discuss technical features, and describe procedural and clinical outcomes.

\section{Methods}

This was a retrospective review of the neurointerventional database from 5 comprehensive stroke centers for patients identified to have a transient ischemic attack or stroke involving the vascular territory of the CaW who ultimately underwent CAS. Patients with cryptogenic stroke etiology according to TOAST classification were included (online suppl. material; see www.karger.com/doi/10.1159/000489710 for all online suppl. material). The study period and contribution breakdown per institution are displayed in the online supplementary material.

CaW was defined by the presence of a shelf-like/linear, smooth filling defect in the posterior aspect of the carotid bulb without superimposed atherosclerotic changes diagnosed by CT angiography (CTA) and confirmed with conventional angiography. All cases were centrally reviewed by a fellowship-trained neurointerventionist. The degree of stenosis was estimated by the NASCET criteria via CTA.

Demographic information, preprocedural care, procedural variables, and clinical outcomes were collected. Follow-up imaging details were collected from radiology reports. The preliminary experience of one of the centers with CaW stenting has previously been partially published [6]. Continuous variables are reported as median (IQR); categorical variables are reported as proportions.

\section{Results}

\section{Baseline Characteristics}

Twenty-four consecutive patients with stented symptomatic CaWs were identified. The qualifying event was a stroke in $20(83 \%)$ and transient ischemic attack in 4 (17\%). Their median age was 47 years (IQR, 41-61) years, 14 (58\%) were female, and 17 (71\%) blacks. Nine (37\%) patients had hypertension, $4(16 \%)$ hyperlipidemia, $0(0 \%)$ atrial fibrillation, 4 $(16 \%)$ diabetes, and $1(4 \%)$ active smoking.

Seven $(29 \%)$ of the patients had recurrent events previous to stent placement (5/7 were taking at least one antiplatelet agent at the time of recurrence). The median NIHSS score at presentation was 12 (IQR 2-16) and the median ASPECTS score was 8 (IQR 7-8). Five (21\%) patients were found to have thrombus superimposed to the CaW; all of them were treated medically and all were confirmed to have resolution prior to stenting. The degree of stenosis generated by the CaW was $0 \%$ (range $0-11 \%$ ). Eleven (46\%) received intravenous thrombolysis and 13 (54\%) thrombectomy (100\% modified TICI 2b-3). No parenchymal hematomas were observed. No patient with CaW was sent for surgical resection within the study period; all revascularized patients were treated endovascularly.

\section{Carotid Artery Stenting}

All patients were stented on dual antiplatelets (aspirin and clopidogrel [20] or ticagrelor [4]) at a median of 9 days (IQR 4-35) after the last vascular event (Fig. 1-3). All cases were 

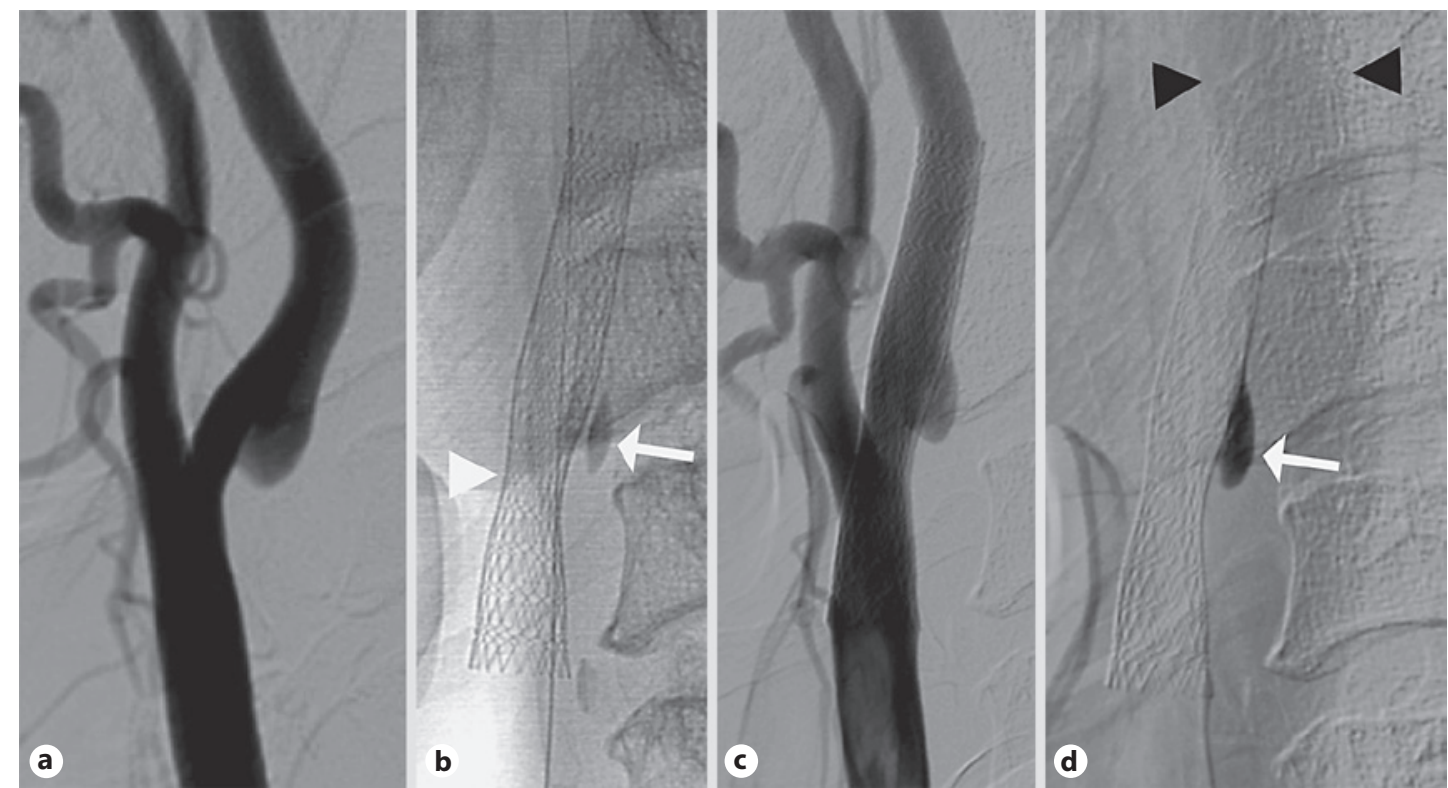

Fig. 1. A 57-year-old man with hypertension and middle cerebral artery stroke. a Lateral digital subtraction angiography of the common carotid artery indicating the CaW. b Fluoroscopy after closed-cell stenting depicting minimal residual waist (white arrowhead) and contrast stagnation within the web pocket (arrow). c Early arterial angiography following stenting. d Venous phase (black arrowheads delineating the margins of the internal jugular vein) with trapping of contrast within the excluded web pocket.
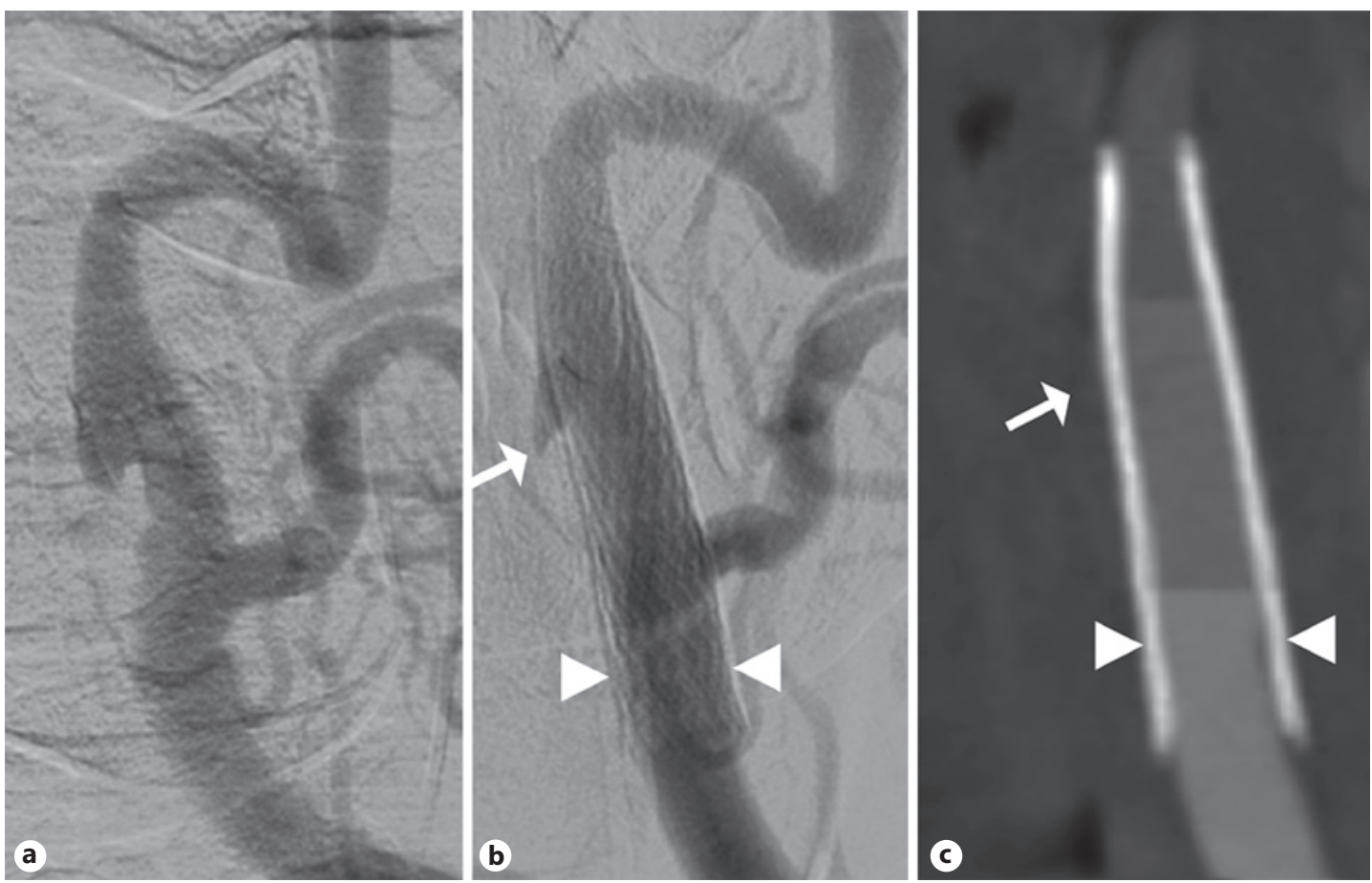

Fig. 2. A 61-year-old woman with no risk factors and anterior circulation stroke. a Left anterior oblique conventional angiography indicating the CaW. b Post-stent (arrowheads: stent margins) angiography demonstrating the remodeling of the CaW pocket (arrow). c A 21-month post-stent CTA angiography showing endoluminal reconstruction (arrow: resolution of the CaW pocket; arrowheads: stent margins). 

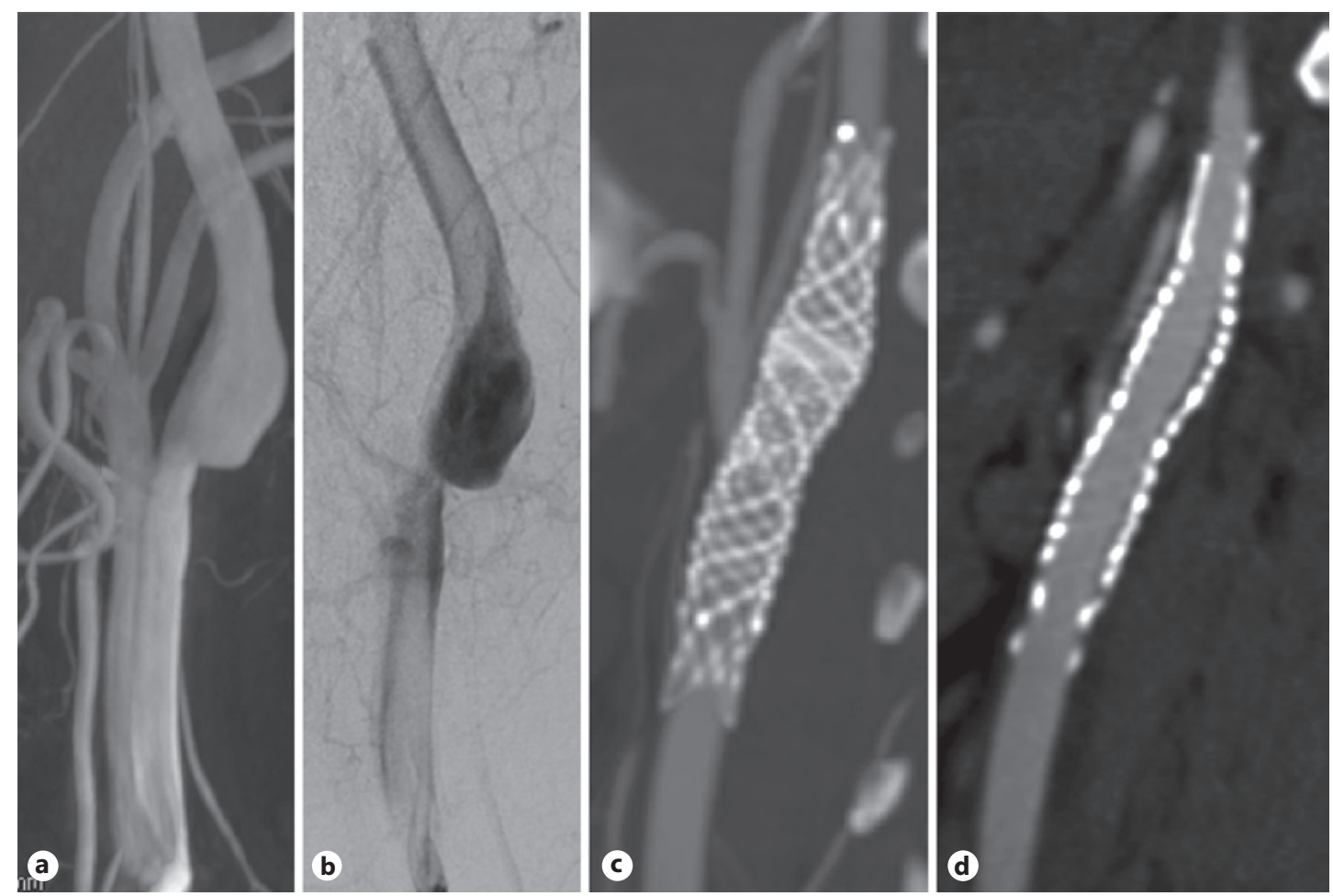

Fig. 3. A 35-year-old man with no risk factors and anterior circulation stroke. a 3D rotational conventional angiogram of the common carotid artery depicting the web. b Mid-arterial phase of the common carotid artery subtraction angiogram. c Maximum intensity projection. d Source image of a 19-month CT angiogram follow-up indicating vessel remodeling with no stenosis.

heparinized to achieve an activated clotting time of 200-300 s. Embolic protection was used in 22 cases (91\%) (Spider FX-Medtronic [ $n=19]$, Emboshield NAV6-Abbott Vascular [ $n=2]$, Mo.Ma-Medtronic $[n=1]$ ). In 3 patients, 2 overlapping stents were used attempting to optimize the vascular remodeling, while in the remaining patients, 1 stent was placed. No cases had preprocedural balloon angioplasty. Closed-cell stents were used in 18 (75\%) of the cases (XACT-Abbott Vascular [ $n=16]$, Wallstent-Medtronic [ $n=1]$, Casper-Microvention $[n$ $=1]$ ) and open-cell stents were used in $6(25 \%)$ (Acculink- Abbott Vascular $[n=5]$ ). No cases required balloon angioplasty for residual stenosis. In 2 patients, despite no residual stenosis, angioplasty was performed in an attempt to improve the stent apposition against the wall without significant change in the stent configuration. No periprocedural adverse events occurred with the exception of 2 cases of asymptomatic hypotension/bradycardia (1 requiring 24-h vasopressors). Dual antiplatelets were maintained for at least 3 months, followed by monotherapy. One patient developed a genitourinary hemorrhage requiring discontinuation of one antiplatelet at 2 months.

\section{Clinical and Radiological Outcomes}

A modified Rankin Scale score of 0-2 (independence) at 90 days was observed in 22 patients (91\%). Twenty-three patients have available follow-up data. No new cerebrovascular events occurred at a median follow-up of 12 months (IQR 3-19). Vascular imaging was performed in all patients (ultrasound in 18 [78\%] and CTA in 5 [22\%]) at a median of 10 months (IQR 3-18) and was uniformly unremarkable. 


\section{Discussion}

We report the results of a multicenter experience in the treatment of CaWs with CAS demonstrating technical feasibility, safety and pilot data on efficacy.

CaW represents an intimal variant of fibromuscular dysplasia. The particular morphology of CaW leads to blood stasis in the rostral aspect of the lesion, which leads to thrombosis, as previously documented by histology, CTA, and conventional angiography [2, 6-8]. Superimposed thrombosis has been reported in $29-40 \%$ of patients with CaW-related strokes and leads to cerebral embolism $[2,6]$.

The optimal treatment strategy for patients with symptomatic CaW remains uncertain. Previous studies have demonstrated that conservative therapy with antiplatelets is associated with relatively high recurrent stroke risks. Patients with CaWs on antiplatelet monotherapy were described to have a $30 \%(6 / 20)$ recurrent ischemic event rate (median time to recurrence 12 months) in a study from Martinique [1]. A Canadian study has demonstrated that 5/7 (71\%) CaW patients with recurrent strokes were on antiplatelets [2]. A study from the US has reported that 5/24 (21\%) patients with symptomatic CaW had recurrent cerebrovascular events while on antiplatelet therapy ( 2 of them being on a dual-antiplatelet regimen) [6]. Considering the proposed mechanism of blood stasis over the CaW and the apparent suboptimal response to antiplatelets [2,6], anticoagulants may be effective. However, CaWs are composed of fibrotic tissue and positive remodeling has never been reported. Therefore, a short-term aggressive antithrombotic regimen (dual antiplatelets or anticoagulation) followed by monotherapy may not be effective considering that the risk of stroke may not significantly decrease over time. Life-long anticoagulation with warfarin (or novel anticoagulants) may be reasonable; however, taking into account that patients with symptomatic CaW are typically young, the cumulative risk of life-long therapy needs to be considered.

In this scenario, revascularization could constitute a reasonable/viable option. Surgical resection for CaW was originally described in 1968 and has historically been used for the treatment of webs over the following decades [1, 2, 7, 9-11]. Although scarce, the data indicates that surgery is safe and potentially effective. Considering the lack of surgical planes, surgery typically involves piecemeal or segmental resection with patching.

The stent construct generates immediate luminal reconstruction with flow reorientation/ diversion. This is followed by endothelialization and, similar to cerebral aneurysms treated with flow-diverting stents or carotid bulb atherosclerotic ulcers, the excluded area (CaW pocket) resolves over time likely due to laminar thrombosis and vascular remodeling $[12,13]$. Scarce literature regarding stenting for symptomatic CaWs is available. Lenck et al. [4] has reported the first 2 patients that underwent uneventful stenting for CaW. Elmokadem et al. [3] reported another 2 treated patients: 1 experienced an iatrogenic midcervical internal carotid dissection which was successfully treated with another stent. Martin-Perez et al. [5] has described 1 patient with symptomatic CaW that was uneventfully stented. Our group has described a preliminary single-institution series of 16 stented CaW patients [6]. All procedures were uneventful but technical details were not provided and clinical and radiological follow-up was limited. Here we present a larger multicenter series corroborating earlier findings of feasibility, safety, and potential efficacy in preventing recurrent strokes. The median clinical follow-up time of all the aforementioned reports is 11.5 months (IQR 3-21). Technically, the procedure is substantially less complex as compared to stenting for atherosclerotic stenosis considering that the lumen is typically preserved (straightforward to cross the lesion) and that pre- and post-stent angioplasty (thromboembolic-prone steps) are not necessary.

The present results should be cautiously analyzed. Although high stroke recurrence rates in symptomatic CaW have been consistently demonstrated in observational studies, many uncertainties remain. Despite the risks of recurrent events on antiplatelets, the role of anti- 
coagulation as compared to revascularization is not known. Although reasonable, the potential benefits of the use of distal protection devices for CaW stenting is not clear. Considering the apparently benign natural history, stenting of asymptomatic CaW should not be pursued. Taking into account that the patients with symptomatic $\mathrm{CaW}$ being medically treated were not included in the present study, selection bias is possible.

\section{Conclusions}

Stenting for symptomatic CaW appears to be a safe and minimally invasive alternative to surgical resection. Further studies are warranted.

\section{Disclosure Statement}

D.C.H., J.A.G., S.K., A.M., D.Y., B.G., W.L., A.E.H., A.-L.D., S.R., C.L., and G.P.: no relevant disclosures. A.E.H.: consulting/speaking: Medtronic, Stryker, Penumbra, Microvention, GE Healthcare, Genentech; proctoring: Medtronic,Microvention.R.G.N.:Stryker-Neurovascular(Trevo-2\&DAWN/TrialPI), Covidien(SWIFT\&SWIFTPRIME/Steering Committee, STAR Trial/Core Lab), Penumbra (3-D Separator Trial/Executive Committee).

\section{Funding Sources}

No funding was received.

\section{References}

1 Joux J, Chausson N, Jeannin S, Saint-Vil M, Mejdoubi M, Hennequin JL, Deschamps L, Smadja D, Olindo S: Carotid-bulb atypical fibromuscular dysplasia in young Afro-Caribbean patients with stroke. Stroke 2014;45: 3711-3713.

2 Choi PM, Singh D, Trivedi A, Qazi E, George D, Wong J, Demchuk AM, Goyal M, Hill MD, Menon BK: Carotid webs and recurrent ischemic strokes in the era of CT angiography. AJNR Am J Neuroradiol 2015;36:2134-2139.

3 Elmokadem AH, Ansari SA, Sangha R, Prabhakaran S, Shaibani A, Hurley MC: Neurointerventional management of carotid webs associated with recurrent and acute cerebral ischemic syndromes. Interv Neuroradiol 2016; 22:432-437.

4 Lenck S, Labeyrie MA, Saint-Maurice JP, Tarlov N, Houdart E: Diaphragms of the carotid and vertebral arteries: an under-diagnosed cause of ischaemic stroke. Eur J Neurol 2014;21:586-593.

5 Martinez-Perez R, Lownie SP, Pandey SK, Boulton MR: Stent placement for carotid web. World Neurosurg 2017;98:879.e9-879.e11.

6 Haussen DC, Grossberg JA, Bouslama M, Pradilla G, Belagaje S, Bianchi N, Allen JW, Frankel M, Nogueira RG: Carotid web (intimal fibromuscular dysplasia) has high stroke recurrence risk and is amenable to stenting. Stroke 2017;48:3134-3137.

7 Kubis N, Von Langsdorff D, Petitjean C, Brouland JP, Guichard JP, Chapot R, Mikol J, Woimant F: Thrombotic carotid megabulb: fibromuscular dysplasia, septae, and ischemic stroke. Neurology 1999;52:883-886.

8 Boesen ME, Eswaradass PV, Singh D, Mitha AP, Goyal M, Frayne R, Menon BK: MR imaging of carotid webs. Neuroradiology 2017;59:361-365.

9 Sajedi PI, Gonzalez JN, Cronin CA, Kouo T, Steven A, Zhuo J, Thompson O, Castellani R, Kittner SJ, Gandhi D, Raghavan P: Carotid bulb webs as a cause of "cryptogenic" ischemic stroke. AJNR Am J Neuroradiol 2017;38: 1399-1404.

10 So EL, Toole JF, Moody DM, Challa VR: Cerebral embolism from septal fibromuscular dysplasia of the common carotid artery. Ann Neurol 1979;6:75-78.

11 Rainer WG, Cramer GG, Newby JP, Clarke JP: Fibromuscular hyperplasia of the carotid artery causing positional cerebral ischemia. Ann Surg 1968;167:444-446.

12 Walcott BP, Stapleton CJ, Choudhri O, Patel AB: Flow diversion for the treatment of intracranial aneurysms. JAMA Neurol 2016;73:1002-1008.

13 Sadikin C, Teng MM, Yeh CC, Chang FC, Luo CB: Morphological changes of ulcerative plaque in patients received carotid angioplasty and stenting (CAS). Eur J Radiol 2008;65:434-441. 